\title{
Improving hypertension control through a collaboration between an academic medical center and a chain community pharmacy
}

\author{
Sarah E. Vordenberg Pharm.D., MPH ${ }^{1}$ | Valerie Lindell Pharm.D. ${ }^{2}$ |

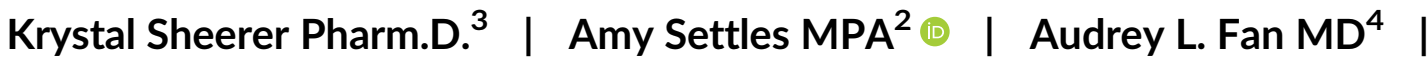 \\ David C. Serlin $M^{5}$ | Ebony Parker-Featherstone $M^{5,6}$ | \\ Steven J. Bernstein MD, MPH ${ }^{7,8} \mid$ Hae M. Choe Pharm.D. ${ }^{1,2}$ (๑)

\footnotetext{
${ }^{1}$ College of Pharmacy, University of Michigan, Ann Arbor, Michigan

${ }^{2}$ Pharmacy Innovations and Partnerships, Michigan Medicine, Ann Arbor, Michigan

${ }^{3}$ Pharmacy, Meijer Inc, Vicksburg, Michigan

${ }^{4}$ Internal Medicine, Michigan Medicine, Northville, Michigan

${ }^{5}$ Family Medicine, Michigan Medicine, Ann Arbor, Michigan

${ }^{6}$ Obstetrics and Gynecology, Ann Arbor, Michigan

${ }^{7}$ Internal Medicine and Center for Clinical Management Research, Michigan Medicine, Ann Arbor, Michigan

${ }^{8}$ VA Ann Arbor Healthcare System, Ann Arbor, Michigan
}

\section{Correspondence}

Hae M. Choe, University of Michigan Health System - Pharmacy Innovations and Partnerships, 1301 Catherine St, SPC 5693 6312 Medical Science I, Ann Arbor, Michigan 48109.

Email: haemi@med.umich.edu

\section{Abstract}

Introduction: Approximately one-third of adults in the United States have hypertension (HTN), leading to increased morbidity and mortality.

Objectives: This quality improvement intervention was designed to improve HTN control among community-dwelling adults through collaboration between patientcentered medical homes (PCMH) within an academic medical center and chain community pharmacies.

Methods: Four PCMH sites in Ann Arbor, Michigan that were in close proximity to two Meijer pharmacies participated in this study between September 2016 and March 2017, which compared HTN outcomes for patients seen at two community pharmacies where the pharmacists received training on HTN management for patients who received usual care at their $\mathrm{PCMH}$. The primary outcome was percent of patients who met their blood pressure (BP) goal of either $<140 / 90 \mathrm{mmHg}$ or $<150 / 90 \mathrm{mmHg}$ compared with matched controls who received usual care at the PCMH. Secondary outcomes included number of medication recommendations made, percent of recommendations accepted by the primary care provider (PCP), and patient satisfaction.

Results: Patients who received care at the community pharmacy $(n=155)$ had a higher rate of $\mathrm{BP}$ control at 3 months than matched controls $(61.8 \%$ vs $47.7 \%$, $P=0.013)$. A total of 29 medication recommendations were made by community pharmacists and 26 were accepted by the PCP. Nearly $95 \%$ of patients rated the care 
they received as excellent or very good and over 95\% stated that they would recommend the pharmacist at the Meijer pharmacy to their family and friends.

Conclusion: Patients who received HTN management services as part of a collaboration between an academic medical center and chain community pharmacy were significantly more likely to have controlled BP at 3 months compared with matched controls who received standard care. This model shows promise as being a strategy to expand access to care for patients while being mutually beneficial for community pharmacies and health systems.

\section{KEYWORDS}

community pharmacy, hypertension, pharmacist, pharmacy

\section{1 | INTRODUCTION}

Approximately one-third of adults in the United States have hypertension (HTN), leading to increased morbidity and mortality, particularly from cardiovascular and kidney diseases. ${ }^{1}$ It is estimated that HTN is the primary or contributing cause in 362000 deaths and it leads to nearly 39 million physician office visits annually. ${ }^{2-4}$ Pharmacologic and nonpharmacologic treatment strategies exist to lower the burden of elevated blood pressure (BP). In the general population in the United States, $17.3 \%$ of people with HTN are unaware of their diagnosis and therefore remain untreated. ${ }^{1}$ Furthermore, $48.2 \%$ of adults with diagnosed HTN continue to have uncontrolled BP and $37 \%$ of people do not take their prescribed antihypertensive medications. ${ }^{1,5}$ Health system leaders at Michigan Medicine (MM), an academic medical center, reviewed Healthcare Effectiveness Data and Information Set (HEDIS) measures and identified that metrics were not being met related to BP management for adults and were charged with developing strategies to improve the process for identifying, treating, and monitoring BP.

A previous study implemented the use of a multidisciplinary team consisting of medical assistants, clerical staff, physicians, and an ambulatory care clinical pharmacist to improve BP control among community-dwelling adults. ${ }^{6}$ Process changes included visual and action-oriented high BP prompts, a collaborative practice agreement, medication intensification protocol, and a home BP monitoring machine loan program. These changes increased the percent of patients meeting BP targets from $53.6 \%$ to $69.3 \%(P<.001){ }^{6}$

While ambulatory care-based pharmacists provide important clinical services, the pharmacist that patients interact with frequently resides in the community setting. Nearly $90 \%$ of Americans live within 5 miles of a community pharmacy, making the community pharmacist the most accessible health care professional. ${ }^{7-9}$ This creates an opportunity for pharmacists to serve as an access point for patients to receive primary care services in the community. Providing nondispensing services at a community pharmacy can increase accessibility for patients by being more convenient than a physician's office and providing opportunities for evening and weekend visits.
Evidence is increasing about the positive impact of community pharmacists on BP management. The Asheville Project demonstrated sustained improvement in BP as a result of community pharmacist education and long-term medication therapy management services. ${ }^{10}$ A randomized trial of 723 patients conducted in 56 community pharmacies in Alberta demonstrated a significant reduction in risk for cardiovascular events, including as a result of systolic BP reduction. ${ }^{11}$ Community pharmacist interventions focused on education, medication management, and lifestyle advice have been associated with a significant reduction in systolic and diastolic BP. ${ }^{12}$ A pilot study demonstrated that community pharmacists trained in $\mathrm{PCMH}$ clinics were as effective at managing HTN as ambulatory care pharmacists at the $\mathrm{PCMH}{ }^{13}$

\section{2 | OBJECTIVE}

The objective of this study was to improve rates of HTN control among community-dwelling adults through collaboration between patient-centered medical homes (PCMH) within an academic medical center and chain community pharmacy.

\section{3 | METHODS}

\section{1 | Setting}

A total of four PCMH sites associated within an academic medical center in Ann Arbor, Michigan that were within close proximity to Meijer pharmacies participated in this study between September 2016 and March 2017. Initially, two PCMH sites were selected to pilot the model and two additional sites were added in February 2017. A total of 16 hours of appointments were available per week between the two Meijer pharmacies. The pharmacies filled approximately 2250 and 2850 prescriptions weekly.

MM paid Meijer pharmacy for HTN management services as improving uncontrolled BP had been selected as a priority for the organization. Based on an agreement, Meijer sent an invoice to MM and was compensated by hours per week, regardless of whether there 
were appointments scheduled or completed. The contracted rate was based on the cost of a pharmacist without advanced training. Meijer pharmacy paid for the pharmacists' time to complete the training and continued to pay for pharmacist benefits. Pharmacists did not participate in the pharmacy workflow during clinic hours; however, they participated in dispensing and nondispensing services before and after the HTN clinic.

\subsection{Community pharmacist participation and training}

Community pharmacists employed at a Meijer pharmacy located at or near a participating location and interested in providing HTN management services were eligible for the position. Interested candidates $(n=5)$ were interviewed by the Meijer pharmacy district manager. Most pharmacists $(n=4)$ had a Doctor of Pharmacy degree The pharmacy district manager playing the role of a patient to assess the pharmacist's HTN management knowledge and motivational interviewing skills. All five pharmacists were trained with the intent that two pharmacists would be dedicated to each Meijer pharmacy plus one additional pharmacist who could provide coverage at both pharmacies, as needed. The district manager who is a licensed pharmacist also completed the training in order to be familiar with the roles and responsibilities of her direct reports.

The district manager referred the pharmacists to the MM Administrative Lead who was responsible for obtaining the curriculum vitaes of the pharmacists and ensuring their licensure with the state (Table A1). The Administrative Lead was the primary point of contact between the pharmacists and $M M$ and oversaw the steps necessary for pharmacists to gain access to the electronic health record (EHR) as well as the nonclinical aspects of training. Pharmacists were required to complete 12 hours of live training regarding how to use the EHR.

The Meijer pharmacists were also supervised by a Clinical Lead who is an ambulatory care pharmacist experienced in HTN management. The Clinical Lead was responsible for providing training regarding HTN management according to the established policies at MM and teaching the pharmacists how to gather and document pertinent information in the EHR. There was approximately 12 hours of clinical training, divided in 3-4 hour weekly sessions.

Pharmacist competence was assessed via multiple choice tests and a review of their documentation and recommendations by the Clinical Lead. At the beginning of training, pharmacists completed a 10-question multiple choice assessment regarding which section of the Subjective, Objective, Assessment, and Plan (SOAP) note to document specific information as well as a 10-question multiple choice assessment regarding clinical and protocol competency. After training was completed and the pharmacist began to provide patient care services, they initially sent their recommendations to the Clinical Lead who helped to building relationships between the physicians and the Meijer pharmacists. After the Clinical Lead agreed with at least $80 \%$ of their recommendations and the pharmacist retook the two multiple choice assessments and scored $100 \%$ on each, they were allowed to send recommendations directly to the primary care provider (PCP) (Table A2).

The health system developed a policy to ensure that all community pharmacists who provided contracted services were supervised by designated MM physicians. One hour bi-monthly videoconferences between the community pharmacists, Clinical Lead, Administrative Lead, and a medical director were held in order to discuss patient cases, review records for completeness and accuracy, review MM guidelines, and discuss new information in the area of HTN management. Two medical directors alternated participation in this meeting.

\section{3 | Intervention}

A protocol was developed to systematically identify patients with high $\mathrm{BP}$ and refer them for follow-up at either their PCMH or Meijer pharmacy (Figure 1). All adults had a BP reading obtained by a medical assistant (MA) as part of the routine intake process when they presented for an appointment at a participating $\mathrm{PCMH}$. If the BP was elevated, the MA rechecked it after at least 5 minutes and if an abnormal reading persisted, the MA attached a standing order for a referral to a pharmacist for BP follow-up in the EHR. If the PCP signed the order, the check-out staff were instructed to schedule the visit. During the first 15 weeks of the intervention, the Administrative Lead also reached out to eligible patients based on recent clinic visit notes in order to build the patient population in the newly created Meijer BP clinics, while helping patients who recently had elevated BP with no follow-up scheduled. This was done as it was anticipated that it would take several weeks for the appointment slots to be filled through referrals from the new program and it was important to give community pharmacists the opportunity to apply the knowledge and skills they had recently developed.

Patients were given the option to follow-up either with a pharmacist at the PCMH or at a Meijer pharmacy within 2-4 weeks. Services were the same at both locations. Check out staff were given a short script about the partnership with Meijer pharmacy including hours of operation for each pharmacy and clarification that there would be no cost. Patients did not need to obtain medications from a Meijer pharmacy in order to participate. Patients called the PCMH if they needed to reschedule the appointment. A program phone number was also created and shared with patients. Clinic staff were asked to call the Administrative Lead if there were any questions regarding the program.

A protocol was developed to standardize the BP visit at the community pharmacy (Table 1). This was based on existing HTN guidelines

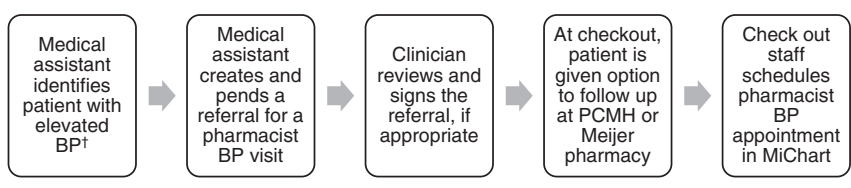

FIGURE 1 Patient identification and referral process at patientcentered medical home (PCMH). BP, blood pressure. 'Systolic blood pressure $\geq 140$ and/or diastolic blood pressure $\geq 90 \mathrm{mmHg}$ 
TAB LE 1 Community pharmacist clinical protocol for blood pressure monitoring

\begin{tabular}{|c|c|c|c|}
\hline $\begin{array}{l}\text { Systolic blood } \\
\text { pressure } \\
(\mathrm{mmHg})\end{array}$ & $\begin{array}{l}\text { Diastolic } \\
\text { blood } \\
\text { pressure } \\
(\mathrm{mmHg})\end{array}$ & Patient symptoms & $\begin{array}{l}\text { Pharmacist } \\
\text { action }\end{array}$ \\
\hline \multicolumn{4}{|l|}{ No symptoms } \\
\hline$<159$ & $<99$ & \multirow[t]{3}{*}{ None } & \multirow{2}{*}{$\begin{array}{l}\text { Note via } \\
\text { in-basket } \\
\text { (high priority) }\end{array}$} \\
\hline $160-200$ & $100-120$ & & \\
\hline 201 or higher & $\begin{array}{l}121 \text { or } \\
\text { higher }\end{array}$ & & $\begin{array}{l}\text { Send to ED/call } \\
911\end{array}$ \\
\hline \multicolumn{4}{|l|}{ Symptoms } \\
\hline Less than 100 & Less than 60 & $\begin{array}{l}\text { Dizziness or } \\
\text { lightheadedness }\end{array}$ & \multirow[t]{2}{*}{$\begin{array}{l}\text { Call clinic or } \\
\text { page PCP }\end{array}$} \\
\hline $140-159$ & $60-99$ & $\begin{array}{l}\text { Shortness of breath, } \\
\text { chest pain, severe } \\
\text { headache }\end{array}$ & \\
\hline $160-200$ & $100-120$ & $\begin{array}{l}\text { Shortness of breath, } \\
\text { chest pain, severe } \\
\text { headache, } \\
\text { symptoms of } \\
\text { stroke, } \\
\text { encephalopathy } \\
\text { (nausea, vomiting, } \\
\text { changes in vision, } \\
\text { decreased } \\
\text { consciousness) }\end{array}$ & \multirow[t]{2}{*}{$\begin{array}{l}\text { Send to } \\
\text { emergency } \\
\text { department } \\
\text { or call } 911\end{array}$} \\
\hline 201 or higher & $\begin{array}{l}121 \text { or } \\
\text { higher }\end{array}$ & $\begin{array}{c}\text { Regardless of } \\
\text { symptoms }\end{array}$ & \\
\hline
\end{tabular}

Note: If the systolic and diastolic blood pressures fall into two different categories, the pharmacist should take the action associated with the higher category (eg, patient with no symptoms with a blood pressure of 182/122 mmHg - send to emergency department or call 911). Abbreviations: ED, emergency department; PCP, primary care physician.

at MM Upon arrival at a Meijer pharmacy, the patient was checked in for their appointment via the EHR by the community pharmacist. All appointments were scheduled for 30 minutes, with new patient appointments generally requiring the full time and return visits typically lasting less than 30 minutes. The original structure allowed for 30 minutes in between each appointment for completing other tasks, namely visit documentation in the EHR. However, the structure was modified to instead give pharmacists 30 minutes before and after the clinic shift due to the increased efficiency of the pharmacists to complete visit documentation. It also allowed for a model that was more scalable from the retail pharmacy perspective, as it lessens the time that the pharmacist spends on documentation.

During the visit, the pharmacist gathered information from the patient related to (1) their lifestyle, such as diet (including sodium and caffeine intake), physical activity, smoking status, alcohol use, and stress; (2) current and previous medication use, tolerability, cost concerns, and adherence; (3) pertinent review of systems such as chest pain, shortness of breath, edema, and pain; and (4) recent home BP values. The patient's BP was collected using the BpTRU machine
(BpTRU Medical Devices, Coquitlam, BC, Canada), which is an automated oscillometric device that obtains six readings 1 to 5 minutes apart, discards the first reading, and averages the remaining five readings. ${ }^{14}$ This device was selected as it is more accurate than taking the average of three manual BP readings in the office setting and as accurate as 24 hours ambulatory BP monitoring (ABPM) or ABPM daytime averages. ${ }^{15,16}$ BP was obtained manually if determined necessary by the pharmacist or requested by the patient. During visits, pharmacists validated home BP cuffs, worked with patients to set diet and physical activity goals, made recommendations to either start, stop, or titrate doses of antihypertensive medications up or down, and recommended basic metabolic panels. Educational materials were handed out at the discretion of the pharmacist.

Information about the visit, including recommendations regarding proposed medication changes, were communicated to the PCP via a standardized template in the EHR sent as high priority the same day as the visit. If there was no acknowledgement within 72 business hours, a second encounter with the same message was routed to the PCP. Pharmacists subsequently called the clinic if there was no response in 24-48 business hours of the second attempt in order to make the medication recommendation to either the PCP or staff member who had the ability to get in contact with the PCP the same day. In the event that the PCP was still unable to be reached, the pharmacist contacted the Meijer Program MM Clinical Lead who was able to page the PCP. If the pharmacist received an automatic message within the EHR indicating that the PCP was out of the office, the Meijer pharmacist called the PCMH clinic and asked the nurse to triage the message to the appropriate covering clinician.

The pharmacist scheduled a follow-up visit with the patient between 2-4 weeks later, depending on whether the BP was controlled or uncontrolled during the visit. If the patient's BP was controlled for two consecutive visits, the patient was discharged back to the PCP.

\section{4 | Data collection and analysis}

Patients who received BP management at a Meijer pharmacy (cases) and the $\mathrm{PCMH}$ (controls) were matched on the basis of age, sex, and BP goal. These criteria were selected based on the information that was readily available about each patient. Any case $(n=2)$ who was not able to be matched was excluded from the analysis comparing systolic and diastolic BP. However, all cases were included in the overall analysis to determine the percent of patients at goal. The percent of patients meeting their BP goal was compared as opposed to reduction in $\mathrm{BP}$, as this aligns with how data is analyzed when looking at HEDIS measures. BP goals were based on Eighth Joint National Committee (JNC 8) Guidelines. ${ }^{17}$ The BP goal was less than $140 / 90 \mathrm{mmHg}$ for patients less than 60 years old and those with diabetes mellitus or chronic kidney disease. The goal was less than $150 / 90 \mathrm{mmHg}$ for patients 60 years or older without diabetes mellitus or chronic kidney disease. The index visit BP, defined as the $\mathrm{BP}$ recorded in the EHR at the PCP appointment, was compared with the last BP recorded in the EHR at 3 months. If the patient did not 
have any follow-up appointments, the BP from the index visit was used in the analysis as this aligns with how data is used when evaluating HEDIS measures. Process measures related to the Meijer pharmacy program included number of referrals, scheduled and completed visits, no show rate, number of medication changes recommended and accepted by the PCP, and patient satisfaction. This study was deemed not regulated by the Institutional Review Board of the University of Michigan Medical School (IRBMED).

Frequency distributions and descriptive statistics were used. Comparison of baseline and 3 months systolic and diastolic BP between patients who received care at a Meijer pharmacy (cases) and $\mathrm{PCMH}$ (controls) were compared using a $t$ test. Rates of BP control were compared using a $\chi^{2}$ test. Each patient was handed a paper survey to complete at the end of their visit at a Meijer pharmacy. The 14-item survey was developed based on an existing patient survey focused on a different topic. Most questions used a three-point Likert scale (yes, definitely; yes, somewhat; and no). The pharmacist left the room and the patient was instructed to place their survey in a locked box upon completion. An administrative assistant was the only person with access to the box and would go to the stores once a month to gather the surveys. Patient satisfaction surveys were collected on an ongoing basis and analyzed for this manuscript through March 2017. Analysis was conducted using Microsoft Excel 2016.

\section{4 | RESULTS}

A total of 200 referrals for BP management services were made during the study period. A total of 33 patients (16.5\%) were identified as part of the central outreach process by the Administrative Lead and the remaining referrals were made by the PCMHs. The median number of referrals was 2.6 per PCMH per week.

The majority of scheduled visits at Meijer pharmacies were for new patient appointments $(\mathrm{n}=205,78.2 \%$ ) and the remaining appointments were for return visits ( $n=57,21.8 \%$ ). The number of new patient appointments scheduled exceeded the number of referrals as patients who did not present for their new patient appointment could schedule an additional new patient appointment. The majority of referred patients ( $n=157 / 200$, 78.5\%) completed their new patient appointment. Over three-fourths of scheduled appointments were completed ( $n=203 / 262,77.5 \%$ ) with no statistically significant difference in the percentage of completed visits for new patient and return visits ( $n=157 / 205,76.6 \%$ and $n=46 / 57,80.7 \%$, respectively). The overall no show rate was $22.5 \%(n=59 / 262)$.

A total of 155 of the 157 unique patients were matched with controls. Baseline and 3 months systolic and diastolic BP was compared (Table 2). At 3 months, $61.8 \%$ of cases met their BP goal compared with $47.7 \%$ of controls $(P=.013$ ) (Table 3 . One-half $(n=12 / 24)$ of the medication recommendations for patients with uncontrolled BP were to add a medication with the remaining recommendations to increase the dose of an existing medication. Most medication recommendations that were made were accepted by the PCP ( $n=26 / 29,89.7 \%$ ) (Table 4). The most common reasons for not making a medication
TABLE 2 Mean baseline and three-month systolic and diastolic blood pressure among cases and controls

\begin{tabular}{|c|c|c|c|}
\hline Time & Controls $(n=155)$ & Cases $(n=155)$ & $P$ value \\
\hline \multicolumn{4}{|c|}{ Systolic blood pressure } \\
\hline Baseline & 153.6 & 150.0 & .029 \\
\hline Three months & 142.0 & 136.5 & .007 \\
\hline \multicolumn{4}{|c|}{ Diastolic blood pressure } \\
\hline Baseline & 85.5 & 86.4 & .400 \\
\hline Three months & 80.5 & 79.0 & .235 \\
\hline
\end{tabular}

TABLE 3 Number and percent of patients reaching blood pressure goal at 3 months

\begin{tabular}{|llll|}
\hline Time & Controls $\mathrm{n}(\%)$ & Cases $\mathrm{n}(\%)$ & P value \\
\hline At goal & $74(47.7)$ & $97(61.8)$ & .013 \\
\hline Not at goal & $81(52.3)$ & $60(38.2)$ & \\
\hline
\end{tabular}

TABLE 4 Medication changes among visits in which patients had controlled or uncontrolled blood pressure

\begin{tabular}{|c|c|c|c|}
\hline & $\begin{array}{l}\text { Uncontrolled } \\
\text { BP }(n=93)\end{array}$ & $\begin{array}{l}\text { Controlled } \\
\text { BP } \\
(n=108)\end{array}$ & $\begin{array}{l}\text { Total } \\
(n=201)\end{array}$ \\
\hline $\begin{array}{l}\text { Number of medication } \\
\text { recommendations } \\
\text { made to PCP }\end{array}$ & 24 & 5 & 29 \\
\hline $\begin{array}{l}\text { Percentage of visits } \\
\text { with medication } \\
\text { recommendation }\end{array}$ & 25.8 & 4.6 & 14.4 \\
\hline $\begin{array}{l}\text { Medication } \\
\text { recommendations } \\
\text { accepted by PCP }\end{array}$ & 21 & 5 & 26 \\
\hline $\begin{array}{l}\text { Percentage of } \\
\text { medication } \\
\text { recommendations } \\
\text { accepted by PCP }\end{array}$ & 87.5 & 100 & 89.7 \\
\hline
\end{tabular}

Abbreviations: BP, blood pressure; PCP, primary care provider.

recommendation when a patient had uncontrolled BP was because the patient was focusing on lifestyle modifications ( $n=27$ ), adherence issues were addressed ( $n=16)$, or the BP was improving $(n=9)$. The reason for medication recommendations during visits with controlled BP included decreasing the dose for patient safety purposes $(n=2)$, switching antihypertensive medications $(n=2)$, and changing the timing of the administration to promote adherence $(n=1)$; all recommendations were accepted by the PCP.

Patients who completed the survey $(n=169 / 203$, 83.3\%) were highly satisfied with the care they received and would recommend the pharmacist to their family and friends. The care provided was generally rated as excellent ( $n=119 / 163,73.0 \%$ ) or very good 
( $n=41 / 16325.2 \%$ ). Examples of feedback that patients provided included "I trust this pharmacist to help me achieve better health," "This was very convenient... The pharmacist was very knowledgeable and I felt confident with the suggestions given," and "The information among with his friendly persona made me feel relaxed. I will take heed to the information about improving my health."

\section{5 | DISCUSSION}

Nearly $14 \%$ more patients who received HTN management services at the community pharmacy met their BP goal compared with patients receiving standard care at the $\mathrm{PCMH}$. Medication recommendations were generally accepted by the PCP. Patients reported being highly satisfied with the service. This is significant as it has been proposed that elimination of HTN could reduce death due to cardiovascular disease by $30 \%$ for men and $38 \%$ for women. ${ }^{18}$

Pharmacists are increasingly being recognized for their ability to provide cost-effective HTN management that improves patient outcomes. ${ }^{19-25}$ The optimal strategy for incorporating pharmacists continues to be studied; however, the necessity of team-based care has been established and there is growing evidence that community pharmacist integration is supported within $\mathrm{PCMHs}{ }^{26,27}$ Extending care into the community allows patients to engage with high quality health care services in a convenient setting. This may provide an opportunity to address persistent HTN-related health disparities.

One of the key elements was to provide training to the community pharmacists. The training model described in this study is more feasible and offers increased opportunities for expansion compared with the immersion program in which the community pharmacist is trained at the PCMH. ${ }^{13}$ Access to the patient's full EHR by the community pharmacist was important to foster bi-directional communication between pharmacists and the referring physicians. Community pharmacist access to the EHR continues to be sparsely reported in the literature. ${ }^{28-30}$ Finally, dedicated time for scheduled patient appointments at the community pharmacy was crucial to the success of this partnership.

The primary limitation to this study is that it represents the work of one academic medical center in collaboration with one chain community pharmacy, and a small number of patients participated. Further work is necessary to determine how to implement this intervention at different institutions with varying organizational structures, opportunities, and barriers. Secondly, the patient population may not represent the general population. Limited characteristic data was collected from individuals and as a result it was not possible to describe the patient population who benefited from this intervention. Additionally, patients self-selected into the community pharmacy intervention and therefore assigning patients to go to a community pharmacy may not lead to the same results. Systolic BP was lower among controls; however, the average BP reduction was higher than the cases. The rate at which PCPs signed the orders for HTN management services needs to be studied. Furthermore, we did not study persistence of lifestyle changes, BP medication adherence, and BP control after patients have reached their BP goal and discontinued follow-up with the service. Studying the sustainability of the clinical service model and exploration about whether and how this program may address health disparities is necessary. Further evaluation of this program is underway in collaboration with the Centers for Disease Control and Prevention to address some of these limitations. ${ }^{31}$

\section{6 | CONCLUSION}

Patients who received HTN management services as part of a collaboration between an academic medical center and chain community pharmacy were significantly more likely to have controlled BP at 3 months compared with matched controls who received usual care (61.8\% vs $47.7 \%, P=.013$ ). Nearly $95 \%$ of patients rated the care they received as excellent or very good and over $95 \%$ stated that they would recommend the pharmacist at the Meijer pharmacy to their family and friends. This model shows promise as being a strategy to expand access to care for patients while being mutually beneficial for community pharmacies and health systems.

\section{CONFLICTS OF INTEREST}

The authors declare no conflicts of interest.

\section{ORCID}

Amy Settles (D) https://orcid.org/0000-0002-4020-2577

Hae M. Choe (iD) https://orcid.org/0000-0002-4642-3864

\section{REFERENCES}

1. Nwankwo T, Yoon SS, Burt V, Gu Q. Hypertension among adults in the United States: National Health and Nutrition Examination Survey, 2011-2012. NCHS Data Brief. 2013;133:1-8.

2. U.S. Preventive Services Task Force. Screening for high BP in adults: U.S. Preventive Services Task Force recommendation statement. Ann Intern Med. 2015;163:778-786.

3. National ambulatory medical care survey: 2010 summary tables [cited 2019 May 14]. Available from: www.cdc.gov/nchs/data/ahcd/namcs summary/2010_namcs_web_tables.pdf.

4. National hospital ambulatory medical care survey: 2011 outpatient department summary tables [cited 2019 May 14]. Available from: www.cdc.gov/nchs/data/ahcd/nhamcs_outpatient/2011_opd_web tables.pdf.

5. HealthyPeople.gov. Heart disease and stroke: Objectives [cited 2019 May 14]. Available from: www.healthypeople.gov/2020/topicsobjectives/topic/heart-disease-and-stroke/objectives.

6. Choe HM, Bernstein SJ, Cooke D, Stutz D, Standiford C. Using a multidisciplinary team and clinical redesign to improve blood pressure control in patients with diabetes. Manage Health Care. 2008;17(3): 227-233.

7. National Center for Chronic Disease Prevention and Health Promotion. A program guide for public health: Partnering with the pharmacist in the prevention and control of chronic diseases. 2012 [cited 2019 June 19]. Available from: www.cdc.gov/dhdsp/programs/spha/ docs/pharmacist_guide.pdf. 
8. National Association of Chain Drug Stores. Re: Health Care Workshop, Project No. P131207 [cited 2019 May 14]. Available from: www.nacds.org/ceo/2014/0508/supplemental_comments.pdf.

9. Kelling SE. Exploring accessibility of community pharmacy services. Innov Pharm. 2015;6(3):210.

10. Bunting BA, Smith BH, Sutherland SE. The Asheville project: Clinical and economic outcomes of a community-based long-term medication therapy management program for hypertension and dyslipidemia. J Am Pharm Assoc. 2008;48(1):23-31.

11. Tsuyuki RT, Hamarneh YN, Jones CA, Hemmelgarn BR. The effectiveness of pharmacist interventions on cardiovascular risk: The multicenter randomized controlled RxEACH trial. J Am Coll Cardiol. 2016;67 (24):2846-2854.

12. Cheema E, Sutcliffe $P$, Singer DR. The impact of interventions by pharmacists in community pharmacies on control of hypertension: $A$ systematic review and meta-analysis of randomized controlled trials. Br J Clin Pharmacol. 2014;78(6):1238-1247.

13. Farris KB, Mitrzyk BM, Batra $P$, et al. Linking the patient-centered medical home to community pharmacy via an innovative pharmacist care model. J Am Pharm Assoc. 2019;59(1):70-78.

14. FAQ. BpTRU: Automated non-invasive blood pressure monitor [cited 2016 Feb 02]. Available from: www.bptru.com/faq/.

15. Beckett L, Godwin M. The BpTRU automatic blood pressure monitor compared to 24 hour ambulatory blood pressure monitoring in the assessment of blood pressure in patients with hypertension. BMC Cardiovasc Disord. 2005;5(18):1-6.

16. Godwin M, Birtwhistle R, Delva D, et al. Manual and automated office measurements in relation to awake ambulatory blood pressure monitoring. Fam Pract. 2011;28:110-117.

17. James PA, Oparil S, Carter BL, et al. Evidence-based guideline for the management of high blood pressure in adults: Report from the panel members appointed to the eighth Joint National Committee (JNC 8). JAMA. 2014;311(5):507-520.

18. Benjamin EJ, Virani SS, Callaway CW, et al. Heart disease and stroke statistics-2018 update: A report from the American Heart Association. Circulation. 2018;137:e67-e492.

19. Centers for Disease Control and Prevention. Using the pharmacists' patient care process to manage high blood pressure: A resource guide for pharmacists. Atlanta, GA: Centers for Disease Control and Prevention, U.S. Department of Health and Human Services, 2016.

20. Isetts BJ, Buffington DE, Carter BL, Smith M, Polgreen LA, James PA. Evaluation of pharmacists' work in a physician-pharmacist collaborative model for the management of hypertension. Pharmacotherapy. 2016;36(4):374-384.

21. Di Palo K, Kish T. The role of the pharmacist in hypertension management. Curr Opin Cardiol. 2018;33(4):382-387.
22. Stanton-Robinson C, Al-Jumaili AA, Jackson A, et al. Evaluation of community pharmacist-provided telephone interventions to improve adherence to hypertension and diabetes medications. J Am Pharm Assoc. 2018;58(4S):S120-S124.

23. Margolis KL, Asche SE, Dehmer SP, et al. Long-term outcomes of the effects of home blood pressure telemonitoring and pharmacist management of blood pressure among adults with uncontrolled hypertension: Follow-up of a cluster randomized clinical trial. JAMA Netw Open. 2018;1(5):e181617.

24. Dehmer SP, Maciosek MV, Trower NK, et al. Economic evaluation of the home blood pressure telemonitoring and pharmacist case management to control hypertension trial. J Am Coll Clin Pharm. 2018;1(1):21-30.

25. Beran M, Asche SE, Bergdall AR, et al. Key components of success in a randomized trial of blood pressure telemonitoring with medication therapy management. J Am Pharm Assoc. 2018;58(6): 614-621.

26. Luder HR, Shannon P, Kirby J, Frede SM. Community pharmacist collaboration with a patient-centered medical home: Establishment of a patient-centered medical neighborhood and payment model. J Am Pharm Assoc. 2018;58(1):44-50.

27. Bates KA, Stafford RA, Teeter BS, Diemer T, Thomas JL, Curran GM. Pharmacist-provided services and community pharmacist integration into a patient-centered medical home: A qualitative study of primary care clinic staff perceptions. J Am Pharm Assoc. 2019;59:S6-S11. https://doi.org/10.1016/j.japh.2019.03.019.

28. Keller ME, Kelling SE, Cornelius DC, Oni HA, Bright DR. Enhancing practice efficiency and patient care by sharing electronic health records. Perspect Health Inf Manage. 2015;12:1b.

29. Hohmeier KC, Spivey CA, Boldin S, Moore TB, Chisholm-Burns M. Implementation of a health information exchange into community pharmacy workflow. J Am Pharm Assoc. 2017;57(5):608-615.

30. Nelson SD, Poikonen J, Reese T, Halta DE, Weir C. The pharmacist and the EHR. J Am Med Inform Assoc. 2017;24(1):193-197.

31. Michigan Medicine and Meijer Pharmacy Program. Field Notes. Centers for Disease Control and Prevention [cited 2019 May 14]. Available from: www.cdc.gov/dhdsp/docs/Michigan_Medicine_Field-Notes-508.pdf.

How to cite this article: Vordenberg SE, Lindell V, Sheerer K, et al. Improving hypertension control through a collaboration between an academic medical center and a chain community pharmacy. J Am Coll Clin Pharm. 2019;2:357-365. https://doi. org/10.1002/jac5.1158 
TAB LE A1 Community blood pressure center on-boarding checklist

Administrative Lead Responsibilities

- Verify current pharmacist license

- Verification of Health Insurance Portability and Accountability Act (HIPAA) training

- Obtain copy of curriculum vitae or resume

- Request MiChart (Epic) access

- Schedule ambulatory pharmacist MiChart training

- Schedule check in/check out training

- Provide training on BpTru device

- Provide training on marking patients as having arrived for their visit in MiChart

Clinical Lead Responsibilities

Michigan Medicine hypertension guidelines, medications, and management training

- Michigan Medicine hypertension protocol

- Michigan Medicine clinical hypertension guidelines

- Hypertension cases presentation

- Hypertension updates in guidelines presentation

- Community pharmacist blood pressure reading protocol

- Physician outreach procedure

- Managing delays in patient care

- Managing resistance/reluctance in patient care

MiChart ambulatory clinical pharmacist training

- Navigation training: labs, vitals, previous medications, notes

- Hypertension in-clinic encounter workflow

- Telephone encounter workflow

Ambulatory clinical pharmacist training

- Community blood pressure center workflow

- Clinical protocol competency

- SOAP note competency

- Documentation processes and standards in MiChart

- Manual blood pressure training

- Home blood pressure cuff validation

Abbreviation: SOAP, subjective, objective, assessment, and plan. 
TABLE A2 Community pharmacist competency evaluation

\begin{tabular}{|c|c|c|c|}
\hline Item & Description & \multicolumn{2}{|c|}{$\begin{array}{l}\text { Performance Evaluation (date/initial } \\
\text { each entry if met required item): Circle } Y \\
\text { for Yes, } N \text { for No }\end{array}$} \\
\hline $\begin{array}{l}\text { Completed training prior to conducting Meijer } \\
\text { Pharmacy HTN visits }\end{array}$ & $\begin{array}{l}\text { - Training checklist completed and initialed where } \\
\text { necessary by Michigan Medicine Clinical and } \\
\text { Administrative Leads }\end{array}$ & $\mathrm{Y} / \mathrm{N}$ & $\mathrm{Y} / \mathrm{N}$ \\
\hline $\begin{array}{l}\text { Clinical Lead no longer sending extensive }{ }^{a} \text { weekly } \\
\text { emails regarding note documentation } \\
\text { improvement/feedback }\end{array}$ & $\begin{array}{l}\text { - Segregation of information into appropriate } \\
\text { note sections - subjective, objective, } \\
\text { assessment, and plan (SOAP) } \\
\text { - Clarity of verbiage } \\
\text { - Following clinical/guideline protocols }\end{array}$ & $\mathrm{Y} / \mathrm{N}$ & $\mathrm{Y} / \mathrm{N}$ \\
\hline $\begin{array}{l}\text { Clinical Lead no longer sending extensive }{ }^{a} \text { weekly } \\
\text { emails regarding patient care follow-up or } \\
\text { outstanding items }\end{array}$ & $\begin{array}{l}\text { - Following physician outreach protocol } \\
\text { - Pending necessary recommended orders - basic } \\
\text { metabolic panel and medication } \\
\text { - Completing patient outreach } \\
\text { Documented and time stamped telephone } \\
\text { call outreach notifying patient of medication } \\
\text { and/or lab recommendation }\end{array}$ & $\mathrm{Y} / \mathrm{N}$ & $\mathrm{Y} / \mathrm{N}$ \\
\hline $\begin{array}{l}\text { Clinical Lead agrees with recent }{ }^{\mathrm{b}} \text { medication/lab } \\
\text { recommendations } \geq 80 \% \text { of the time (agrees } \\
\text { with last } 4 / 5 \text { recommendations) }\end{array}$ & $\begin{array}{l}\text { - Medication choice and rationale } \\
\text { - Basic metabolic panel timing obtainment }\end{array}$ & $\mathrm{Y} / \mathrm{N}$ & $\mathrm{Y} / \mathrm{N}$ \\
\hline Competencies $100 \%$ score & $\begin{array}{l}\text { - Two competencies provided during training } \\
\text { (clinical protocol; SOAP note) }\end{array}$ & $\mathrm{Y} / \mathrm{N}$ & $\mathrm{Y} / \mathrm{N}$ \\
\hline Ongoing provider proficiency evaluation & $\begin{array}{l}\text { Physician review of } 2-3 \text { patient case encounters } \\
\text { containing medication changes every } 8 \text { months } \\
\circ \text { Occur during bi-monthly videoconference } \\
\text { calls with medical directors }\end{array}$ & $\mathrm{Y} / \mathrm{N}$ & $\mathrm{Y} / \mathrm{N}$ \\
\hline
\end{tabular}

Note: Pharmacists who meet competency standards described below are able to send recommendations directly to Primary Care Provider instead of Clinical Lead for approval.

Abbreviation: HTN, hypertension.

${ }^{a}$ Containing more than three items.

${ }^{\mathrm{b}}$ Last five. 